Review Article

\title{
NMDA Receptor Subunits Change after Synaptic Plasticity Induction and Learning and Memory Acquisition
}

\author{
María Verónica Baez $(\mathbb{D}$, Magalí Cecilia Cercato, and Diana Alicia Jerusalinsky \\ Instituto de Biología Celular y Neurociencia "Prof. E. De Robertis", UBA-CONICET, School of Medicine, University of Buenos Aires, \\ 2155 Paraguay St., 1121 CABA, Argentina
}

Correspondence should be addressed to María Verónica Baez; Mveritobaez@gmail.com and Diana Alicia Jerusalinsky; Djerusal@gmail.com

Received 25 May 2017; Revised 17 July 2017; Accepted 4 January 2018; Published 7 March 2018

Academic Editor: Hee J. Chung

Copyright (C) 2018 María Verónica Baez et al. This is an open access article distributed under the Creative Commons Attribution License, which permits unrestricted use, distribution, and reproduction in any medium, provided the original work is properly cited.

\begin{abstract}
NMDA ionotropic glutamate receptors (NMDARs) are crucial in activity-dependent synaptic changes and in learning and memory. NMDARs are composed of two GluN1 essential subunits and two regulatory subunits which define their pharmacological and physiological profile. In CNS structures involved in cognitive functions as the hippocampus and prefrontal cortex, GluN2A and GluN2B are major regulatory subunits; their expression is dynamic and tightly regulated, but little is known about specific changes after plasticity induction or memory acquisition. Data strongly suggest that following appropriate stimulation, there is a rapid increase in surface GluN2A-NMDAR at the postsynapses, attributed to lateral receptor mobilization from adjacent locations. Whenever synaptic plasticity is induced or memory is consolidated, more GluN2A-NMDARs are assembled likely using GluN2A from a local translation and GluN1 from local ER. Later on, NMDARs are mobilized from other pools, and there are de novo syntheses at the neuron soma. Changes in GluN1 or NMDAR levels induced by synaptic plasticity and by spatial memory formation seem to occur in different waves of NMDAR transport/expression/degradation, with a net increase at the postsynaptic side and a rise in expression at both the spine and neuronal soma. This review aims to put together that information and the proposed hypotheses.
\end{abstract}

\section{Introduction}

Learning and memory, as well as synaptic plasticity which is considered their electrophysiological correlate, depend on glutamatergic transmission (reviewed in [1]). AMPA ( $\alpha$-amino-3-hydroxy-5-methyl-4-isoxazolepropionic acid) and NMDA ( $N$-methyl-D-aspartate) ionotropic glutamate receptors (AMPARs and NMDARs, resp.) are crucial in activity-dependent synaptic changes (reviewed in [2-5]). While AMPARs are able to mediate ordinary glutamate neurotransmission, the NMDAR channel is usually blocked by extracellular $\mathrm{Mg}^{2+}$ at cell membrane resting potentials (reviewed in [1]). To achieve effective transmission through NMDARs, they must be unblocked by membrane depolarization through the activation of AMPARs, while at the same time, both glutamate and glycine must bind the receptor. Therefore, NMDARs are considered molecular coincidence detectors of pre- and postsynaptic activities [6].

NMDARs are heterotetramers mainly present not only at the postsynaptic but also at the presynaptic side of glutamatergic synapses in the central nervous system (CNS). NMDARs are composed of 2 GluN1 obligatory subunits encoded by one gene, with eight variants originated by alternative splicing $[7,8]$, and 2 regulatory subunits that contain the glutamate binding site. Those regulatory subunits are encoded by different genes; there are four GluN2 subunits (GluN2A-D), which are codified by four different genes, and two GluN3 subunits (GluN3A and B), which are codified by two different genes $[5,9]$. At least theoretically, there are more than 60 possibilities to combine these subunits. However, only 9 receptor subtypes have been described, which could be classified as diheteromeric $\left(\mathrm{GluN1}_{2}\right.$-GluN2 $\mathrm{A}_{2}, \mathrm{GluN1}_{2}$-GluN2B, $\mathrm{GluN1}_{2}$-GluN2C $\mathrm{C}_{2}$, 
GluN1 2 -GluN2D 2 , and $\mathrm{GluN1}_{2}-\mathrm{GluN}_{3} \mathrm{~A}_{2}$ ) and triheteromeric (GluN1 ${ }_{2}$-GluN2A-GluN2B, GluN1 2 -GluN2B-GluN2C, GluN1 $1_{2}$-GluN2B-GluN2D, and GluN1 2 -GluN2B-GluN3A) (reviewed in $[5,10,11]$ ).

The regulatory subunit composition of NMDARs defines its pharmacological and kinetic properties [3-5, 9, 12]. Expression of regulatory subunits is dynamic and seems to be tightly regulated in time and space $[4,12,13]$. In CNS regions involved in cognitive functions, like the hippocampus and prefrontal cortex (PFC), GluN2A and GluN2B are the major regulatory subunits $[5,13]$.

During prenatal life, NMDARs containing GluN2B subunit (GluN2B-NMDAR) are predominant, being GluN2B the major regulatory subunit expressed along embryonic development in studied mammals [14]. During early postnatal life, there is an increase in GluN2A expression both in transcription and translation, while GluN2B expression appears to remain low and constant. As a consequence, the GluN2A/GluN2B ratio rises up during that period $[15,16]$, known as the "NMDAR developmental switch" (reviewed in $[12,13])$. In Sprague-Dawley rats, a further increase in GluN2A levels appears to occur after that developmental switch, at least in the hippocampus, where the GluN2A/ GluN2B ratio was reported to be higher in hippocampal protein extracts obtained from 6-month-old rats compared to 35-day-old rats [14]. Although there are several reports, little is known about changes in NMDAR expression after plasticity induction or memory acquisition and the putative involved mechanisms and physiological meaning of such modifications. This review aims to put together that information and the proposed hypotheses on those changes.

\section{Changes in NMDAR Expression after Plasticity Induction (Table 1)}

NMDARs participate in physiological plasticity in the nervous system during development, as well as in synaptogenesis and synapse maturation along the whole life. Also, NMDARs are involved in pathological forms of plasticity, as in epilepsy (reviewed in [4]), stroke and hypoxia (reviewed in $[17,18]$ ), and neurodegenerative disorders like Alzheimer's (reviewed in $[5,13,19])$ and Parkinson's disease (reviewed in $[5,13$, $20,21])$. The lack of either NMDARs or some of their subtypes (i.e., knocking down either GluN1 expression or some regulatory subunit) led to different sorts of long-term plasticity (LTP) or depression (LTD) deficits (reviewed in [22-25]). NMDAR-dependent synaptic plasticity could also be affected by stress or corticosterone treatment ([26]), ethanol (reviewed in [27]), and several drug exposure (reviewed in [13]).

Williams et al. [28] had reported that there were several waves of NMDAR subunit rises, after LTP induction by high-frequency stimulation (HFS). Both GluN2A and GluN2B levels increased 20 minutes and $48 \mathrm{~h}$ after LTP induction in DG total extracts, without significant changes $1 \mathrm{~h}$ or $4 \mathrm{~h}$ after stimulation. Later on, it was shown that both GluN1 and GluN2B levels were significantly higher in synaptosomal fractions $20 \mathrm{~min}$ and 48 hours after HFS and that there also was an increase in GluN1 $8 \mathrm{~h}$ after stimulation in total DG homogenates [29]. The authors attributed the $48 \mathrm{~h}$ rise in GluN1 mainly to an increase in NMDARs at the cell surface, though not at the synaptic membrane, as GluN1 was not significantly higher in the postsynaptic density fraction [30].

On the other hand, Bellone and Nicoll [31] have shown that LTP induction by HFS, in fresh hippocampal slices from newborn mice, led to a rapid shift from GluN2B-NMDAR to GluN2A-NMDAR mediated currents. Those changes take place in milliseconds to seconds and were attributed to simultaneous lateral mobilization of GluN2A-NMDAR along the membrane from extrasynaptic sites and internalization of GluN2B-NMDAR. Furthermore, Barria and Malinow [32] reported a GluN2A-NMDAR increase in dendritic spines after LTP induction in organotypic cultures of hippocampal slices from neonatal rats (immunocytochemistry). Accordingly, Grosshans et al. [33] showed that GluN1 and GluN2A levels were higher in western blots of synaptosomal fractions, correlated with lower levels in these same subunits in nonsynaptic fractions, 30 minutes after plasticity induction in hippocampal slices from 6- to 8-week-old rats. These works suggested strongly that the GluN2A-NMDAR rise at the synapses could be due to mobilization of preassembled NMDARs from nonsynaptic pools $[32,33]$.

We have analyzed NMDAR subunits level following induction of LTP by theta burst stimulation (TBS), in young adult rat hippocampus fresh slices. In slices where potentiation seemed to be effective up to 30 minutes post-TBS (at least as short-term potentiation), GluN1, GluN2A, and GluN2B total levels remained similar to control levels [34]. It has to be taken into account that, while Grosshans et al. determined NMDAR subunits in synaptosomes [35], we used total hippocampal homogenates [34], where exchanges between subcellular fractions could be masked.

We have also reported that 70 minutes after effective LTP induction by TBS, in fresh hippocampal slices from young adult rats, there was an increase in GluN1 and GluN2A levels, while GluN2B remained constant, as determined by western blot in total hippocampus extracts. The rise in both GluN1 and GluN2A subunits only occurred when an effective long-term synaptic plasticity (lasting more than 60 minutes) was established [34]. It must be emphasized that in TBS-stimulated slices where potentiation failed, GluN1, GluN2A, and GluN2B levels remained similar to controls (without stimulation) at 30 and 70 minutes after TBS.

In line with those results, Udagawa et al. [36] showed in primary neuron cultures stimulated by NMDA that GluN2A mRNA localizes in dendrites, where it is inefficiently translated due to its short poly(A) tail. However, after NMDAR stimulation, Gld2 (a poly(A) polymerase) catalyzes poly $(\mathrm{A})$ addition to GluN2A mRNA, leading to translational enhancement of GluN2A mRNA and to an increase in GluN2A levels (measured by western blot), 30 minutes after NMDAR stimulation. The same authors reported that GluN1, but not GluN2B, also increased in dendrites at the same time as GluN2A, but independently of poly(A) polymerization. Furthermore, Swanger et al. [37] have shown that GluN2A local translation and surface expression initiate immediately after LTP induction 


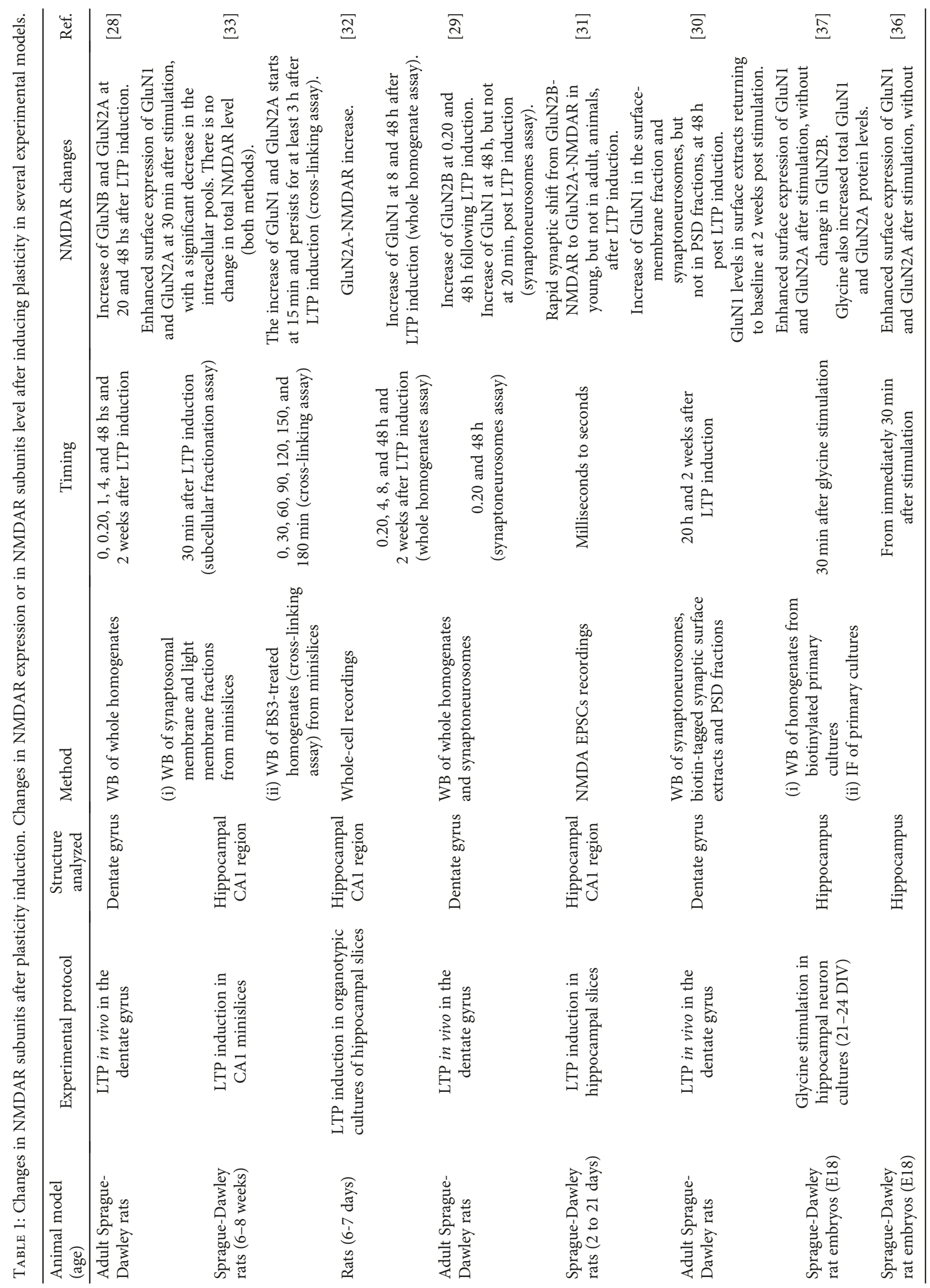




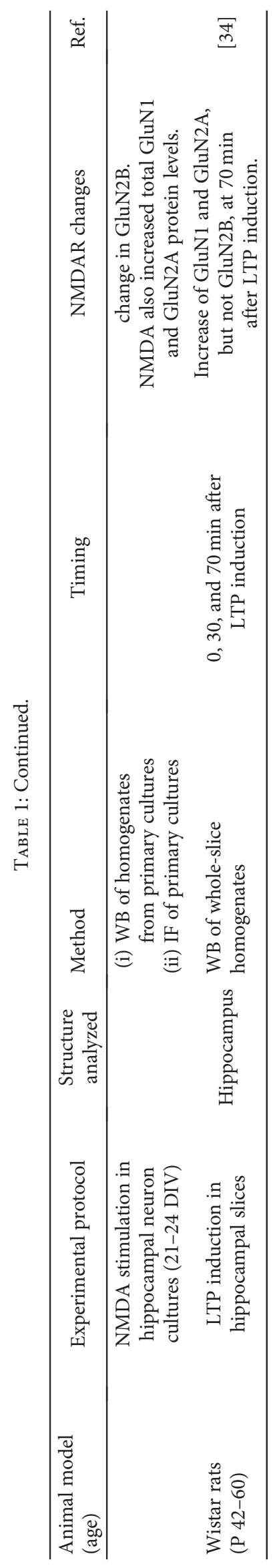


and continue at least for the following 30 minutes (the last time point they analyzed).

In a similar model, we have found that there are equivalent changes in NMDAR subunits even after 30 min poststimulation [34, 38]. GluN2A puncta was significantly increased in cultured hippocampal neurons 30 minutes poststimulation by $\mathrm{KCl}$ pulses and it continued rising up to 75 minutes. Thereafter, GluN2A puncta decreases, reaching control levels 90 minutes after stimulation. On the other hand, GluN1 also starts to increase at 30 minutes, reaching a maximum at 75 minutes. GluN1, as GluN2A puncta, did not differ from nonstimulated controls 90 minutes poststimulus [38].

Some reports have suggested that NMDAR levels could be degraded by different mechanisms. For instance, the binding of GluN1 to Fbx2 (a F box protein associated to the E2 ligase complex), labels this particular NMDAR as proteasome target [39]. Nevertheless, further investigation is necessary in order to clarify NMDAR subunit degradation mechanism after plasticity induction. In line with this, Corbel et al. [40] have shown that GluN2A translation is regulated by miR19 along development, decreasing GluN2A expression during early development.

Concerning possible mechanisms involved in the rise of NMDAR subunits, CHX treatment either totally [37] or partially blocked $[34,38]$ the increase in GluN2A subunits at dendrites. However, this (CHX) treatment did not affect GluN1 increase at dendrites, suggesting that GluN2ANMDAR increase could be due to local translation of GluN2A subunit, which would rapidly be assembled with GluN1 subunits retained inside some local ER vesicles [37, 38].

Furthermore, when CHX was previously added to the media, the increase of both GluN1 and GluN2A subunits, which achieves a maximum at 70-75 minutes after plasticity induction, was fully blocked in neuronal bodies (cultured neurons) as well as in hippocampal slice homogenates. On the other hand, actinomycin D (ActD) treatment did not affect either LTP induction in hippocampal slices or GluN2A increase in both slice homogenates and hippocampal neuron cultures. However, ActD treatment blocked GluN1 increase both in slice homogenates after LTP induction and in soma of cultured neurons after $\mathrm{KCl}$ stimulation, indicating that, at least for the GluN1 rise, de novo synthesis was necessary $[34,38]$.

Altogether, the above-reported data strongly suggest that following an appropriate stimulus, there is a rapid increase in surface GluN2A-NMDAR at the postsynaptic side, which is likely due to lateral receptor mobilization from adjacent locations (Figure 1, step 1). Whenever plasticity was effectively induced, more GluN2A-NMDARs would be assembled using GluN2A from local translation and GluN1 retained in local ER (Figure 1, step 2). As more NMDARs are needed at the spines, mobilization from other pools would contribute to fill up these requirements (Figure 1, step 3). It is conceivable that when the different pools were decreasing, some signals activate NMDAR subunit expression at the neuronal soma, which would lead to a transient increase in subunit level there (Figure 1, steps 4-5). Once synaptic plasticity has been established and the postsynaptic side has already been remodeled, the high concentration of NMDARs could result in excitotoxicity. It is feasible that existent GluN2A-NMDAR would be regulated by ubiquitination and degradation by the proteasome and that de novo expression would be regulated, that is, by miRNAs [39, 40], restoring control levels.

\section{NMDAR Expression and Memory Acquisition (Table 2)}

NMDAR subunits increase was also reported to occur in vivo in animal models, after various experiences. Different changes in GluN1, GluN2A, and GluN2B have been reported to occur in several central structures (Table 2). Such differences could be due to the different techniques used to evaluate memory (including different tasks) and to determine NMDAR subunits. Here, we summarize main changes in NMDAR subunits levels after memory acquisition.

In a task classically used for spatial learning in the rat, the hidden platform version of the Morris water maze (MWM), Cavallaro et al. [41] have shown (by microarray followed by qPCR conformation) that GluN1 mRNA expression was downregulated $1 \mathrm{~h}$ after four consecutive training sessions, whereas GluN2A mRNA expression was similar to that in controls. They have also found that it was upregulated when assessed $24 \mathrm{~h}$ after training. However, in rats trained in the same task with a long-term memory paradigm, Zhang et al. [42] found that GluN1 immunofluorescence was increased in CA1 and DG after 10 trials. This last result is similar to that observed after synaptic plasticity induction described above in the previous section [34, 38]. Moreover, Zhang et al. have also shown that no significant difference was found in long-term memory expression for a long-trained (LT) group of animals compared to a short-trained with reinforcement group (SRT). Accordingly, the intensity of GluN1 immunoreactivity in CA1 and dentate gyrus in LT and SRT rats was significantly higher than that in short-trained or control groups. The comparison of both sets of results led to the interpretation that this increase of GluN1 expression in CA1 and dentate gyrus could be involved in spatial longterm memory formation.

We have shown that both GluN1 and GluN2A subunits increased in the hippocampus of 1-, 2-, and 3-month-old Wistar rats following habituation to a new environment (open field (OF) task) [34]. This increase begins after 30 minutes of a 5-minute session in an OF, which leads to habituation, and reaches a maximum at about 70 minutes. Thereafter, GluN1 and GluN2A levels fall down, being similar to controls at about 90 minutes posttraining. This time course was rather similar to that described in vitro for cultured hippocampal neurons [38]. Therefore, both in vitro and in vivo changes reported by others and us are transient, with rather similar time courses and direction. No significant changes were found, in other analyzed structures like amygdala and PFC 70 minutes after habituation to the OF, nor in the hippocampus after testing the rats in the OF $24 \mathrm{~h}$ later $[34,38]$. Also, we described an increase in GluN1 and GluN2A subunits in the rat hippocampus with a similar time course, following the object exposure phase of a two-object recognition task, though not after the different tests; even when a familiar 


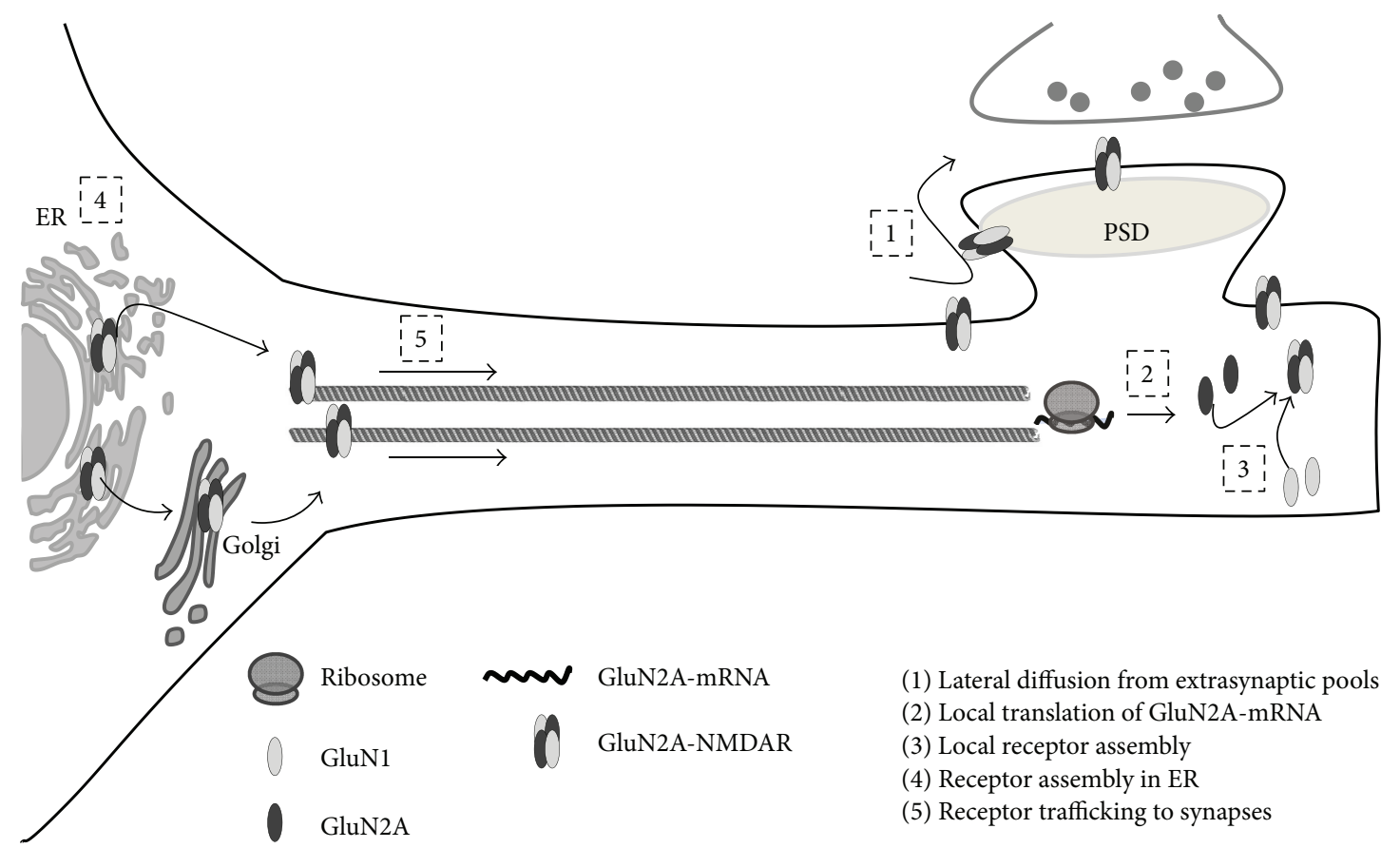

FIGURE 1: Schematic representation of the proposed model for NMDAR localization and expression after plasticity induction. After a stimulus that would elicit long-term plasticity, there is a rapid increase in surface GluN2A-NMDAR at the postsynaptic side, which is likely due to lateral receptor mobilization from adjacent locations (step 1). Whenever plasticity was effectively induced, more GluN2A-NMDARs would be assembled using GluN2A from local translation and GluN1 retained in local ER (step 2). As more NMDARs are needed, mobilization from other pools would contribute to enhance NMDAR expression at synapses (step 3). As nonsynaptic pools decrease, some signals should activate NMDAR subunits expression at the neuronal soma, which would lead to a transient increase in subunits level there (steps 4-5).

object and a new object were presented, the new object was effectively discriminated by the animal [38].

Interestingly, Hepp et al. [43] have recently found in an invertebrate that, although total GluN1 level remained unchanged in crabs after spatial memory acquisition, GluN1 expression at the neuron surface fell down immediately after a 50-minute training. The authors also assessed GluN1 surface expression 3 hours later and found that there was an increase in GluN1 in membrane samples. This increase was also transient, without difference with control level $24 \mathrm{~h}$ later.

NMDAR changes, like a GluN1 increase, were also reported following other spatial tasks like a radial maze and a hole board. Shanmugasundaram et al. [44] have observed, in a synaptosomal fraction extracted $6 \mathrm{~h}$ after training rats in a radial maze along 10 consecutive days, that there was a rise in both GluN1 and GluN2B at the hippocampus and an increase in GluN1 and GluN2A in PFC. The same team also found that there was an increase in GluN1 and GluN2A in the synaptosomal fraction of the dorsal hippocampus, and a later increase in GluN1 and GluN2B in PFC, in rats that were trained in a hole-board along 3 consecutive days and were then stimulated with a weak tetanizing stimulus that would not lead to late-LTP by itself, compared with nontrained though stimulated rats. In spite of the weak stimulation, in such trained rats, the "underthreshold" stimulus led to L-LTP that lasted up to 6 hours [45]. Unfortunately, in this case, it is not known if the raise in NMDAR subunits would take place anyway without the electrical stimulation.
A few works showed that there were changes in NMDAR expression and/or localization using paradigms that are associated with strong emotion. In a step-down inhibitory avoidance of a mild foot-electric shock, Cammarota et al. [46] showed that there was an increase of GluN1 in hippocampal synaptosomal fractions (by western blot), without significant changes in GluN2A or in GluN2B 30 minutes after training; 120 minutes after training, NMDAR subunits level was similar to controls. Mukherjee et al. $[47,48]$ showed that, in P7 to P10 pups, when the developmental switch from GluN2B to GluN2A did not take place yet [12], there was a change in NMDAR as the absolute amount of the essential GluN1 decreased three hours after one training session in an odor preference task. This decrease was observed in synaptosomal fractions of the anterior piriform cortex [47] and in postsynaptic density fractions [48]. However, $24 \mathrm{~h}$ after training, GluN1 level was not significantly different compared to controls, indicating that the modification was transient. Authors also showed that GluN1 downregulation was initiated by mGluR-mediated calcineurin signaling and inferred dephosphorylation and internalization of NMDARs. On the other hand, $24 \mathrm{~h}$ after two trials, there was no significant change in GluN1 level compared to control level.

Changes in NMDAR subunits level have also been described following fear conditioning, Sun et al. [49] have shown that there was a rapid and transient increase in the amount of membrane GluN2B-NMDARs (as both GluN1 and GluN2B increased) in CA1 area, 5 to 10 minutes after a 


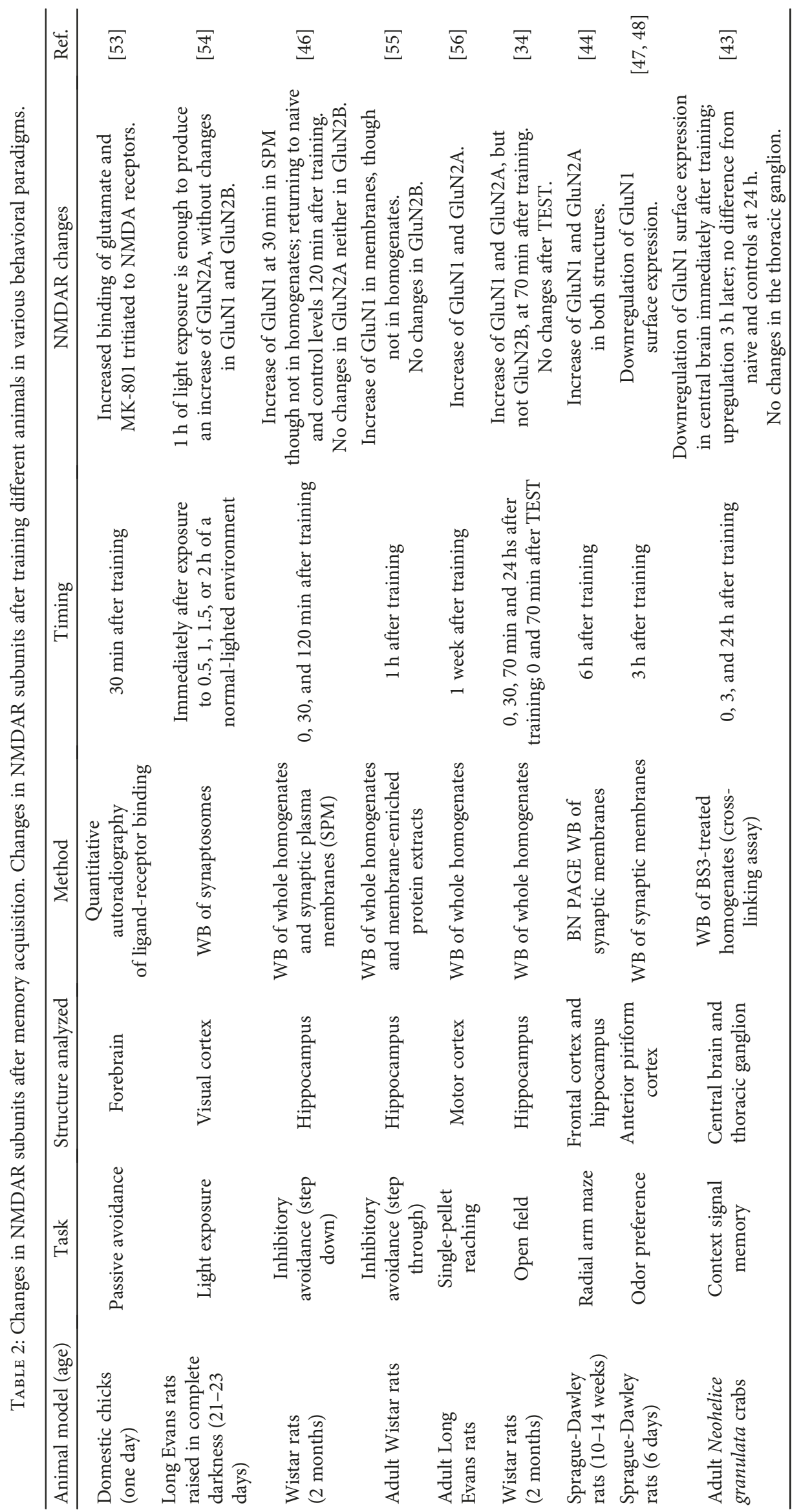




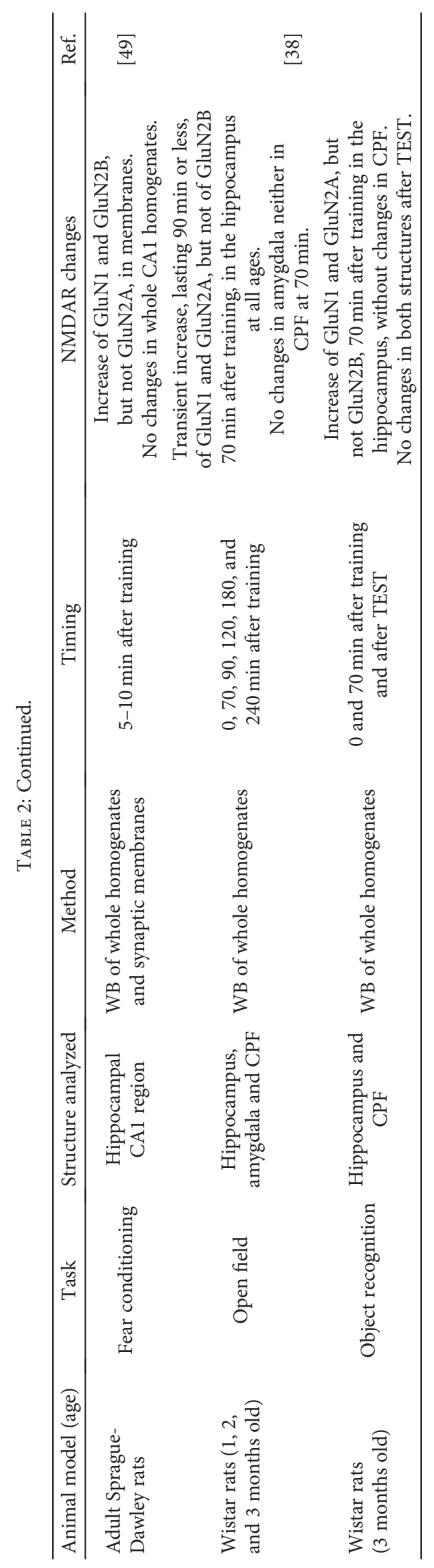


single-trial fear conditioning training. The authors proposed that GluN1 and GluN2B increases depend on GluN2B activation, as it was blocked by GluN2B inhibitors. Accordingly, Sun et al. have also suggested that the reported subunit increase could depend on training strength, as a 5-trial conditioning induced higher subunits levels than a single-trial training [49].

\section{Final Considerations on the Hypotheses Proposed}

NMDAR's central role in synaptic plasticity under physiological conditions is based on its high permeability to calcium ions, as the triggering of both NMDAR-dependent LTP and LTD, at least in the CA1 region of the hippocampus, requires a rise in postsynaptic calcium. But this is also the basis of NMDAR's role in excitotoxic pathological conditions that could lead to neuronal death.

We wonder, for instance, how hippocampal neurons would avoid excitotoxicity after facilitation/potentiation induction through NMDARs, having into account the further increase they seem to undergo for long-term plasticity establishment and long-term memory consolidation. Since the cloning of the different subunits, searching the relationships between NMDAR subtypes and the corresponding functions has been a continuous challenge (reviewed in $[4,12,13,24]$ ). As shown by pharmacological studies, activation of GluN2BNMDARs led to excitotoxic cell death in vitro and in vivo, whereas activation of GluN2A-NMDARs appears neuroprotective [50]. Moreover, GluN2B-NMDARs are associated with pro-death cellular pathways $[51,52]$.

Could an increase in GluN2A, that is, at hippocampal synapses, represent a homeostatic mechanism to normalize synaptic plasticity modifications as to avoid unwanted collateral effects following long-term plasticity?

Altogether, these data could lead to the hypothesis that changes in GluN1 or NMDAR levels induced by synaptic plasticity and by, mainly, spatial memory formation seem to occur in different waves of NMDAR transport/expression/degradation, with an increase in postsynaptic membranes, a rise in local and central expression, followed by degradation and relocalization, and a decrease in expression. This waves could subserve to different synaptic/neuronal functions, depending on the structure, sign, and time period of the molecular/synaptic change.

We propose that the subunits increase from about 20-30 to 75 minutes, which has already disappeared at 90 minutes, after plasticity induction or memory acquisition, could be acting as a check point or a synaptic tag for plasticity establishment or memory consolidation. An increase in the synaptic GluN2A-NMDAR versus GluN2B-NMDAR ratio could act as stabilizer of some synaptic/circuital changes [51], hence leading to stabilize memory consolidation, particularly of spatial representations.

\section{Conflicts of Interest}

The authors declare that they have no conflicts of interest.

\section{Acknowledgments}

The authors acknowledge supporting grants from Universidad de Buenos Aires (UBACYT 20020130100575BA), CONICET (PIP 112201-501001-36CO), and ANPCyTFONCyT (PICT 2013-2221).

\section{References}

[1] E. R. Kandel, J. H. Schwartz, T. M. Jessell, S. A. Siegelbum, and A. J. Hudspeth, Eds., Principles of Neural Science, McGrawHill, New York, NY, USA, 2013.

[2] F. Asztély and B. Gustafsson, "Ionotropic glutamate receptors. Their possible role in the expression of hippocampal synaptic plasticity," Molecular Neurobiology, vol. 12, no. 1, pp. 1-11, 1996.

[3] R. Dingledine, K. Borges, D. Bowie, and S. F. Traynelis, "The glutamate receptor ion channels," Pharmacological Reviews, vol. 51, no. 1, pp. 7-61, 1999.

[4] C. G. Lau and R. S. Zukin, "NMDA receptor trafficking in synaptic plasticity and neuropsychiatric disorders," Nature Reviews Neuroscience, vol. 8, no. 6, pp. 413-426, 2007.

[5] P. Paoletti, C. Bellone, and Q. Zhou, "NMDA receptor subunit diversity: impact on receptor properties, synaptic plasticity and disease," Nature Reviews Neuroscience, vol. 14, no. 6, pp. 383-400, 2013.

[6] P. H. Seeburg, N. Burnashev, G. Köhr, T. Kuner, R. Sprengel, and H. Monyer, "The NMDA receptor channel: molecular design of a coincidence detector," Recent Progress in Hormone Research, vol. 50, pp. 19-34, 1995.

[7] M. C. Paupard, L. K. Friedman, and R. S. Zukin, "Developmental regulation and cell-specific expression of $\mathrm{N}$-methylD-aspartate receptor splice variants in rat hippocampus," Neuroscience, vol. 79, no. 2, pp. 399-409, 1997.

[8] D. Laurie and P. Seeburg, "Regional and developmental heterogeneity in splicing of the rat brain NMDAR1 mRNA," The Journal of Neuroscience, vol. 14, no. 5, 1994.

[9] D. J. Goebel and M. S. Poosch, "NMDA receptor subunit gene expression in the rat brain: a quantitative analysis of endogenous mRNA levels of $\mathrm{NR}_{\text {Com }}$, NR2A, NR2B, NR2C, NR2D and NR3A," Molecular Brain Research, vol. 69, no. 2, pp. 164-170, 1999.

[10] A. Kumar, "NMDA receptor function during senescence: implication on cognitive performance," Frontiers in Neuroscience, vol. 9, p. 473, 2015.

[11] N. A. O’Rourke, N. C. Weiler, K. D. Micheva, and S. J. Smith, "Deep molecular diversity of mammalian synapses: why it matters and how to measure it," Nature Reviews Neuroscience, vol. 13, no. 6, pp. 365-379, 2012.

[12] K. Yashiro and B. D. Philpot, "Regulation of NMDA receptor subunit expression and its implications for LTD, LTP, and metaplasticity," Neuropharmacology, vol. 55, no. 7, pp. 10811094, 2008.

[13] A. Sanz-Clemente, R. A. Nicoll, and K. W. Roche, "Diversity in NMDA receptor composition: many regulators, many consequences," The Neuroscientist, vol. 19, no. 1, pp. 62-75, 2013.

[14] N. Sans, R. S. Petralia, Y. X. Wang, J. Blahos, J. W. Hell, and R. J. Wenthold, "A developmental change in NMDA receptor-associated proteins at hippocampal synapses," The Journal of Neuroscience, vol. 20, no. 3, pp. 1260-1271, 2000. 
[15] H. Hoffmann, T. Gremme, H. Hatt, and K. Gottmann, "Synaptic activity-dependent developmental regulation of NMDA receptor subunit expression in cultured neocortical neurons," Journal of Neurochemistry, vol. 75, no. 4, pp. 1590-1599, 2000.

[16] H. Monyer, N. Burnashev, D. J. Laurie, B. Sakmann, and P. H. Seeburg, "Developmental and regional expression in the rat brain and functional properties of four NMDA receptors," Neuron, vol. 12, no. 3, pp. 529-540, 1994.

[17] T. W. Lai, S. Zhang, and Y. T. Wang, "Excitotoxicity and stroke: identifying novel targets for neuroprotection," Progress in Neurobiology, vol. 115, pp. 157-188, 2014.

[18] E. Shohami and A. Biegon, "Novel approach to the role of NMDA receptors in traumatic brain injury," CNS \& Neurological Disorders - Drug Targets, vol. 13, no. 4, pp. 567-573, 2014.

[19] M. C. Dinamarca, J. A. Ríos, and N. C. Inestrosa, "Postsynaptic receptors for amyloid- $\beta$ oligomers as mediators of neuronal damage in Alzheimer's disease," Frontiers in Physiology, vol. 3, p. 464, 2012.

[20] U. Heresco-Levy, S. Shoham, and D. C. Javitt, "Glycine site agonists of the $\mathrm{N}$-methyl-D-aspartate receptor and Parkinson's disease: a hypothesis," Movement Disorders, vol. 28, no. 4, pp. 419-424, 2013.

[21] L. D. Loopuijt and W. J. Schmidt, "The role of NMDA receptors in the slow neuronal degeneration of Parkinson's disease," Amino Acids, vol. 14, no. 1-3, pp. 17-23, 1998.

[22] D. M. Bannerman, B. Niewoehner, L. Lyon et al., "NMDA receptor subunit NR2A is required for rapidly acquired spatial working memory but not incremental spatial reference memory," The Journal of Neuroscience, vol. 28, no. 14, pp. 36233630, 2008.

[23] R. Place, C. Lykken, Z. Beer et al., "NMDA signaling in CA1 mediates selectively the spatial component of episodic memory," Learning \& Memory, vol. 19, no. 4, pp. 164-169, 2012.

[24] D. M. Bannerman, "Fractionating spatial memory with glutamate receptor subunit-knockout mice," Biochemical Society Transactions, vol. 37, no. 6, pp. 1323-1327, 2009.

[25] O. A. Shipton, O. Paulsen, and O. Paulsen, "GluN2A and GluN2B subunit-containing NMDA receptors in hippocampal plasticity," Philosophical Transactions of the Royal Society B: Biological Sciences, vol. 369, no. 1633, article 20130163, 2013.

[26] Y. C. Tse, R. C. Bagot, J. A. Hutter, A. S. Wong, and T. P. Wong, "Modulation of synaptic plasticity by stress hormone associates with plastic alteration of synaptic NMDA receptor in the adult hippocampus," PLoS One, vol. 6, no. 11, article e27215, 2011.

[27] N. Morisot and D. Ron, "Alcohol-dependent molecular adaptations of the NMDA receptor system," Genes, Brain and Behavior, vol. 16, no. 1, pp. 139-148, 2017.

[28] J. M. Williams, S. E. Mason-Parker, W. C. Abraham, and W. P. Tate, "Biphasic changes in the levels of $N$-methyl-D-aspartate receptor-2 subunits correlate with the induction and persistence of long-term potentiation," Molecular Brain Research, vol. 60, no. 1, pp. 21-27, 1998.

[29] J. M. Williams, D. Guévremont, J. T. T. Kennard, S. E. MasonParker, W. P. Tate, and W. C. Abraham, "Long-term regulation of $n$-methyl-D-aspartate receptor subunits and associated synaptic proteins following hippocampal synaptic plasticity," Neuroscience, vol. 118, no. 4, pp. 1003-1013, 2003.

[30] J. T. T. Kennard, D. Guévremont, S. E. Mason-Parker, W. C. Abraham, and J. M. Williams, "Increased expression, but not postsynaptic localisation, of ionotropic glutamate receptors during the late-phase of long-term potentiation in the dentate gyrus in vivo," Neuropharmacology, vol. 56, no. 1, pp. 66-72, 2009.

[31] C. Bellone and R. A. Nicoll, "Rapid bidirectional switching of synaptic NMDA receptors," Neuron, vol. 55, no. 5, pp. 779785, 2007.

[32] A. Barria and R. Malinow, "Subunit-specific NMDA receptor trafficking to synapses," Neuron, vol. 35, no. 2, pp. 345-353, 2002.

[33] D. R. Grosshans, D. A. Clayton, S. J. Coultrap, and M. D. Browning, "Analysis of glutamate receptor surface expression in acute hippocampal slices," Science's STKE, vol. 2002, article pl8, no. 137, 2002.

[34] M. V. Baez, M. V. Oberholzer, M. C. Cercato, M. Snitcofsky, A. I. Aguirre, and D. A. Jerusalinsky, "NMDA receptor subunits in the adult rat hippocampus undergo similar changes after 5 minutes in an open field and after LTP induction," PLoS One, vol. 8, no. 2, article e55244, 2013.

[35] D. R. Grosshans, D. A. Clayton, S. J. Coultrap, and M. D. Browning, "LTP leads to rapid surface expression of NMDA but not AMPA receptors in adult rat CA1," Nature Neuroscience, vol. 5, no. 1, pp. 27-33, 2002.

[36] T. Udagawa, S. A. Swanger, K. Takeuchi et al., "Bidirectional control of mRNA translation and synaptic plasticity by the cytoplasmic polyadenylation complex," Molecular Cell, vol. 47, no. 2, pp. 253-266, 2012.

[37] S. A. Swanger, Y. A. He, J. D. Richter, and G. J. Bassell, “Dendritic GluN2A synthesis mediates activity-induced NMDA receptor insertion," The Journal of Neuroscience, vol. 33, no. 20, pp. 8898-8908, 2013.

[38] M. C. Cercato, C. A. Vázquez, E. Kornisiuk et al., "GluN1 and GluN2A NMDA receptor subunits increase in the hippocampus during memory consolidation in the rat," Frontiers in Behavioral Neuroscience, vol. 10, p. 242, 2017.

[39] A. Kato, N. Rouach, R. A. Nicoll, and D. S. Bredt, "Activitydependent NMDA receptor degradation mediated by retrotranslocation and ubiquitination," Proceedings of the National Academy of Sciences of the United States of America, vol. 102, no. 15 , pp. 5600-5605, 2005.

[40] C. Corbel, I. Hernandez, B. Wu, and K. S. Kosik, "Developmental attenuation of $\mathrm{N}$-methyl-D-aspartate receptor subunit expression by microRNAs," Neural Development, vol. 10, no. 1, p. 20, 2015.

[41] S. Cavallaro, V. D’Agata, P. Manickam, F. Dufour, and D. L. Alkon, "Memory-specific temporal profiles of gene expression in the hippocampus," Proceedings of the National Academy of Sciences of the United States of America, vol. 99, no. 25, pp. 16279-16284, 2002.

[42] L. Zhang, W. Yu, T.-Z. Han, W. Xie, and Y. Luo, "Changes of NMDA receptor expression in rat hippocampal formation after establishment of spatial memory by different training modes," Sheng Li Xue Bao, vol. 58, no. 5, pp. 442-448, 2006.

[43] Y. Hepp, A. Salles, M. Carbo-Tano, M. E. Pedreira, and R. Freudenthal, "Surface expression of NMDA receptor changes during memory consolidation in the crab Neohelice granulata," Learning \& Memory, vol. 23, no. 8, pp. 427-434, 2016.

[44] B. Shanmugasundaram, A. Sase, A. G. Miklosi et al., "Frontal cortex and hippocampus neurotransmitter receptor complex level parallels spatial memory performance in the radial arm 
maze," Behavioural Brain Research, vol. 289, pp. 157-168, 2015.

[45] S. Subramaniyan, V. Hajali, T. Scherf et al., "Hippocampal receptor complexes paralleling LTP reinforcement in the spatial memory holeboard test in the rat," Behavioural Brain Research, vol. 283, pp. 162-174, 2015.

[46] M. Cammarota, M. L. de Stein, G. Paratcha, L. R. M. Bevilaqua, I. Izquierdo, and J. H. Medina, "Rapid and transient learningassociated increase in NMDA NR1 subunit in the rat hippocampus," Neurochemical Research, vol. 25, no. 5, pp. 567$572,2000$.

[47] B. Mukherjee, G. L. Morrison, C. J. Fontaine, Q. Hou, C. W. Harley, and Q. Yuan, "Unlearning: NMDA receptor-mediated metaplasticity in the anterior piriform cortex following early odor preference training in rats," The Journal of Neuroscience, vol. 34, no. 15, pp. 5143-5151, 2014.

[48] B. Mukherjee, C. W. Harley, and Q. Yuan, "Learning-induced metaplasticity? Associative training for early odor preference learning down-regulates synapse-specific NMDA receptors via mGluR and calcineurin activation," Cerebral Cortex, vol. 27, no. 1, pp. 616-624, 2015.

[49] Y.-Y. Sun, W. Cai, J. Yu et al., "Surface expression of hippocampal NMDA GluN2B receptors regulated by fear conditioning determines its contribution to memory consolidation in adult rats," Scientific Reports, vol. 6, no. 1, article 30743, 2016.

[50] Y. Liu, T. P. Wong, M. Aarts et al., "NMDA receptor subunits have differential roles in mediating excitotoxic neuronal death both in vitro and in vivo," The Journal of Neuroscience, vol. 27, no. 11, pp. 2846-2857, 2007.

[51] C. Taghibiglou, H. G. S. Martin, T. W. Lai et al., "Role of NMDA receptor-dependent activation of SREBP1 in excitotoxic and ischemic neuronal injuries," Nature Medicine, vol. 15, no. 12, pp. 1399-1406, 2009.

[52] W. Tu, X. Xu, L. Peng et al., "DAPK1 interaction with NMDA receptor NR2B subunits mediates brain damage in stroke," Cell, vol. 140, no. 2, pp. 222-234, 2010.

[53] R. J. Steele, M. G. Stewart, and S. P. Rose, "Increases in NMDA receptor binding are specifically related to memory formation for a passive avoidance task in the chick: a quantitative autoradiographic study," Brain Research, vol. 674, no. 2, pp. 352-356, 1995.

[54] E. M. Quinlan, B. D. Philpot, R. L. Huganir, and M. F. Bear, "Rapid, experience-dependent expression of synaptic NMDA receptors in visual cortex in vivo," Nature Neuroscience, vol. 2, no. 4, pp. 352-357, 1999.

[55] S. Moyano, J. Del Río, and D. Frechilla, “Acute and chronic effects of MDMA on molecular mechanisms implicated in memory formation in rat hippocampus: surface expression of CaMKII and NMDA receptor subunits," Pharmacology Biochemistry and Behavior, vol. 82, no. 1, pp. 190-199, 2005.

[56] A. K. Henderson, Q. J. Pittman, and G. C. Teskey, "High frequency stimulation alters motor maps, impairs skilled reaching performance and is accompanied by an upregulation of specific GABA, glutamate and NMDA receptor subunits," Neuroscience, vol. 215, no. 15, pp. 98-113, 2012. 


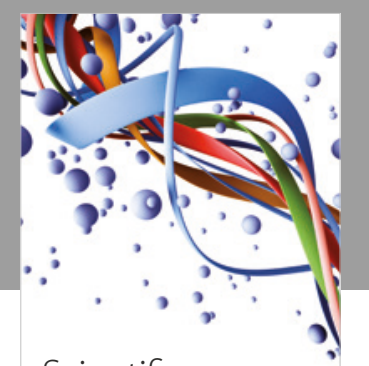

Scientifica
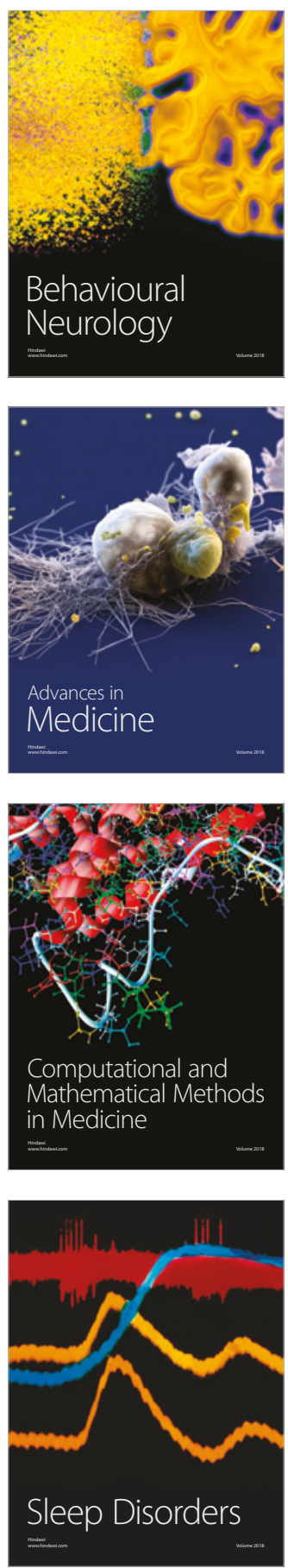

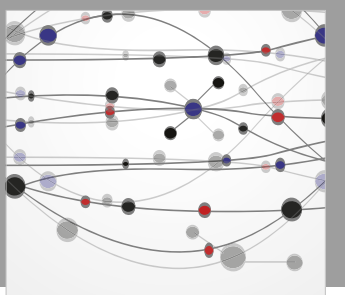

The Scientific World Journal

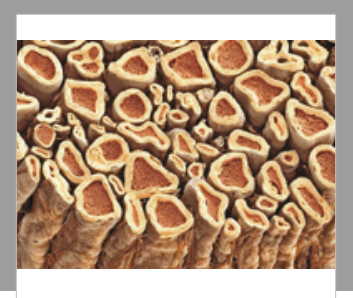

Case Reports in

Neurological Medicine

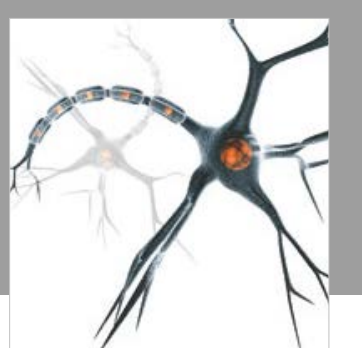

Neural Plasticity

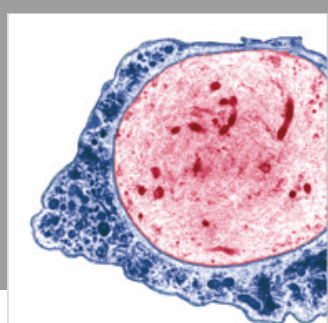

Multiple Sclerosis

International

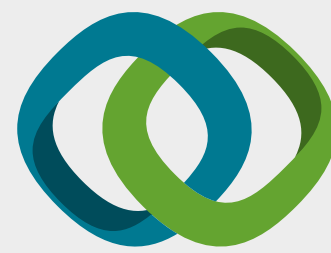

Hindawi

Submit your manuscripts at

www.hindawi.com
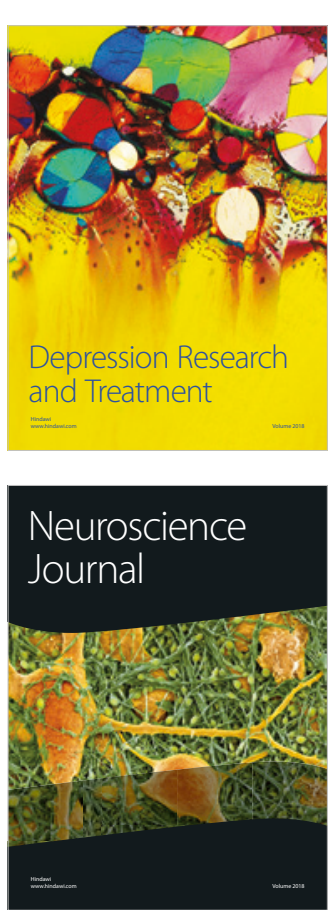

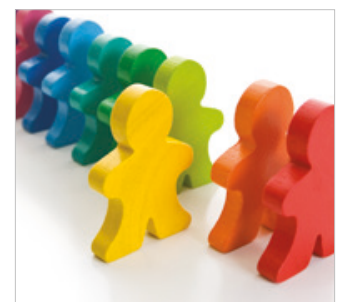

Autism

Research and Treatment
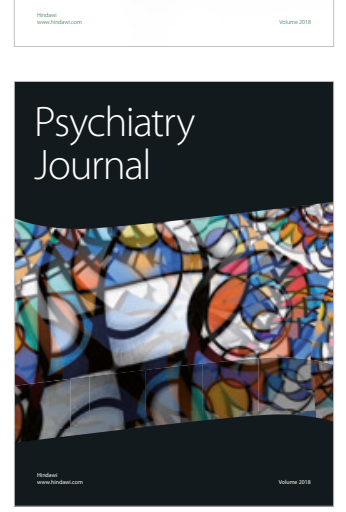
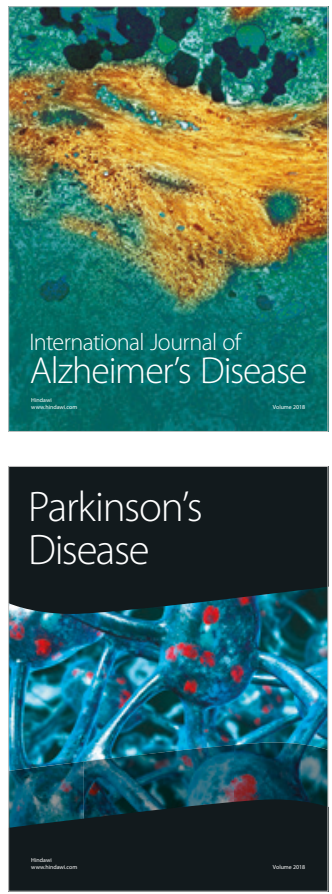
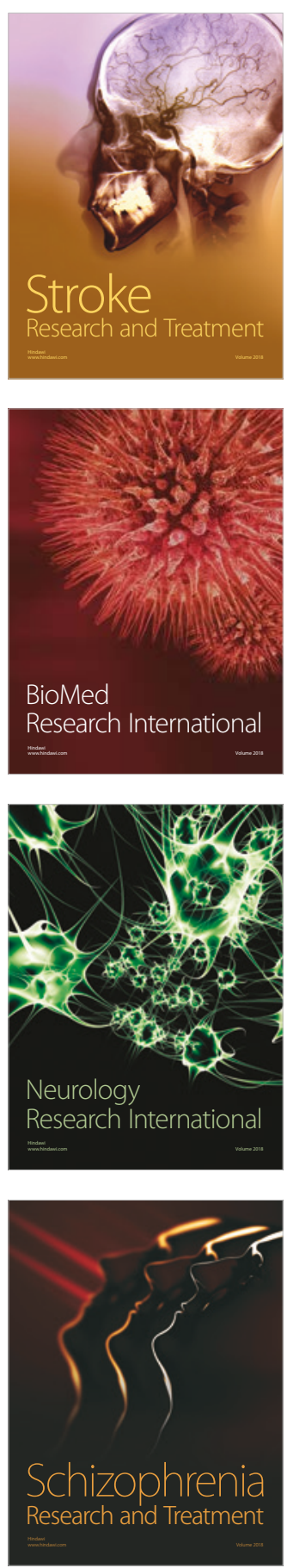